\title{
Comportamento higroscópico do açaí e cupuaçu em pó
}

\author{
Hygroscopic behavior of açaí and cupuaçu powders
}

\author{
Alessandra Eluan da SILVA ${ }^{1}$, Luiza Helena Meller da SILVA², Rosinelson da Silva PENA ${ }^{2 *}$
}

\section{Resumo}

Neste trabalho, isotermas de adsorção e dessorção do açaí (Euterpe oleracea Mart.) e do cupuaçu (Theobroma grandiflorum Schum.) em pó foram determinadas experimentalmente com o auxílio de higrômetro AQUAlab3TE da Decagon nas temperaturas de 15 , 25 e $35{ }^{\circ} \mathrm{C}$. As curvas obtidas apresentaram um comportamento do tipo III, característico de materiais ricos em carboidratos. Os dados experimentais de adsorção e dessorção dos produtos foram ajustados, por análise de regressão não-linear, usando o aplicativo STATISTICA para Windows 5.1B, aos modelos matemáticos de Handerson, Oswin, GAB e BET-modificado. Os modelos de Oswin e GAB foram os que melhor se ajustaram aos dados experimentais de sorção de umidade para o açaí e cupuaçu em pó, respectivamente. Determinou-se ainda a monocamada para os dois produtos e o calor de dessorção para o açaí em pó.

Palavras-chave: isotermas; sorção; modelagem; monocamada; calor de dessorção.

\begin{abstract}
The aim of this work was to evaluate experimentally the adsorption and desorption isotherm of açai (Euterpe oleracea) and cupuaçu (Theobroma grandiflorum) powders using a hygrometer AQUAlab3TE of Decagon. Three levels of temperature $\left(15,25\right.$ and $\left.35^{\circ} \mathrm{C}\right)$ were tested. The type III curves obtained suggest the materials are rich in carbohydrates. Four mathematical models were fitted to the experimental data, Handerson, Oswin, GAB, and modified BET by nonlinear regression analysis using the software STATISTICA for Windows 5.1B. The Oswin and GAB models were better fitted to the açaí and cupuaçu powder sorption data, respectively. The monolayer for açaí and cupuaçu powders was determined. The desorption heat of the açaí powder was also determined.
\end{abstract}

Keywords: isotherm; sorption; modeling; monolayer; desorption heat.

\section{Introdução}

A Região Amazônica possui uma variedade de fruteiras consideradas potenciais e dentre elas se destacam: o açaí (Euterpe oleracea Mart.) e o cupuaçu (Theobroma grandiflorum Schum), devido a seu elevado potencial econômico e valores nutricionais. São utilizados na elaboração de vários produtos alimentícios, desde que se aplique uma tecnologia adequada. De acordo com dados do IBGE (2006) a produção nacional dos frutos de açaí somou, em 2006, 101.341 toneladas, sendo o Estado do Pará o principal produtor do fruto, concentrando $87,4 \%$ desta produção. O cupuaçu, no mesmo ano, teve uma produção de 39.045 toneladas apenas no Estado do Pará. Ambos os frutos são utilizados na fabricação de sucos, doces, geléias e sorvetes, sendo o açaí bastante consumido na Região Norte como "vinho de açaí", podendo ser acompanhado de farinha e açúcar. A principal forma de comercialização do açaí e do cupuaçu é como polpa pasteurizada/congelada, em embalagens de polietileno de $200 \mathrm{~g}$ e $1 \mathrm{~kg}$, e em tambores de $200 \mathrm{~L}$. As duas primeiras formas são utilizadas para a comercialização local e a última, quando o produto é comercializado para fora das fronteiras do estado.

Com o objetivo de difundir esses frutos para os demais estados e até países, é necessária técnica mais elaborada e adequada que mantenha a qualidade dos produtos e aumente a vida-de-prateleira. Um dos grandes problemas no comércio do açaí e do cupuaçu é que são frutos altamente perecíveis, mesmo sob refrigeração. Para contornar esse problema, açaí e cupuaçu estão sendo submetidos a um processo de secagem, obtendo-se produtos desidratados, em condições adequadas de armazenamento.

Assim, torna-se necessário o estudo da relação entre a água e outros componentes de um alimento que defina a sua higroscopicidade; característica essa que exerce influência sobre sua manipulação, processamento e estocagem (LABUZA, 1968).

Uma das ferramentas utilizadas no estudo do comportamento higroscópico de um alimento são as isotermas de adsorção e dessorção de umidade. Entretanto, após o levantamento experimental da isoterma, muitas vezes, se faz necessário a utilização de modelos matemáticos que facilitam a predição de um maior número de pontos partindo de poucos pontos experimentais, devido à limitação de medida dos higrômetros em baixos níveis de atividade de água $\left(\mathrm{a}_{\mathrm{w}}\right)$ e ao rápido desenvolvimento de fungos em altos níveis de $\mathrm{a}_{\mathrm{w}}$ (ROCKLAND, 1957).

Os objetivos deste trabalho foram obter isotermas de adsorção e dessorção de umidade para os produtos comerciais açaí e cupuaçu em pó a 15,25 e $35^{\circ} \mathrm{C}$; testar a aplicabilidade

Recebido para publicação em 11/7/2007

Aceito para publicação em 24/7/2008 (002675)

1 Universidade Federal do Pará - UFPA, CEP 66075-110, Belém - PA, Brasil

${ }^{2}$ Faculdade de Engenharia de Alimentos, Universidade Federal do Pará - UFPA, Belém - PA, Brasil, E-mail: rspena@ufpa.br

${ }^{*}$ A quem a correspondência deve ser enviada 
dos modelos matemáticos de Handerson, Oswin GAB e BETmodificado na predição dos dados de sorção de umidade dos produtos; determinar a monocamada para os produtos e o calor de dessorção para o açaí em pó.

\section{Material e métodos}

\subsection{Matéria-prima}

O açaí e o cupuaçu em pó estudados foram obtidos em estabelecimento comercial da cidade de Belém (PA). Ambas as matérias-primas encontravam-se devidamente desidratadas e armazenadas em embalagens de filme metálico, sob vácuo. De acordo com o fornecedor, a secagem de ambos os produtos foi realizada em spray dryer, sem a adição de coadjuvante.

\subsection{Caracterização físico-química da matéria-prima}

O açaí e o cupuaçu em pó foram caracterizados de acordo com as metodologias da AOAC (1997), sendo determinados: umidade em estufa a $105^{\circ} \mathrm{C}$, até peso constante; proteínas totais, pelo método de Kjedhal, com fator de conversão de nitrogênio para proteína de 6,25; resíduo mineral fixo (cinzas), por calcinação em mufla a $550{ }^{\circ} \mathrm{C}$; teor de gorduras (extrato etéreo), através de extração com éter de petróleo, em extrator Soxhlet; carboidratos, obtidos por diferença.

\subsection{Obtenção das isotermas de sorção}

Para obtenção dos dados de adsorção, amostras dos produtos, com aproximadamente $2 \mathrm{~g}$ do material, foram pesadas em cápsulas plásticas, próprias do equipamento, com auxílio de balança analítica (A\&D Company, Limited modelo HR-200). As amostras foram acondicionadas em dessecador com sílica-gel, sob vácuo, durante 72 horas, à temperatura ambiente $\left(\approx 25^{\circ} \mathrm{C}\right)$, para redução da umidade residual. Em seguida, as amostras foram dispostas em dessecador com água na base, o qual foi levado para a estufa incubadora, nas temperaturas de trabalho $\left(15,25\right.$ e $\left.35^{\circ} \mathrm{C}\right)$, com variação de $\pm 1^{\circ} \mathrm{C}$.

Para obtenção dos dados de dessorção, as mesmas amostras foram dispostas em dessecador com água na base e mantidas por 24 horas nas temperaturas de trabalho, para obtenção do máximo possível de umidade. Em seguida, foram transferidas para dessecador contendo sílica-gel, sendo o conjunto acondicionado em estufa, à temperatura controlada.

Nos dois casos, amostras foram retiradas, em duplicata, em tempos crescentes para determinação da umidade (pseudo-equilíbrio), por diferença de peso, e da atividade de água, em higrômetro AQUAlab 3TE da Decagon. Esta metodologia já foi testada por Guerra, Neves e Pena (2005) e Assunção e Pena (2007) e é utilizada nas pesquisas do grupo de alimentos da Universidade Federal do Pará, tendo apresentado excelente reprodutibilidade.

Foram testados os modelos bi-paramétricos de Handerson e Oswin, e tri-paramétricos de GAB e BET-modificado (Tabela 1), comumente empregados em tal predição. Os ajustes foram realizados por regressão não-linear, com o auxílio do aplicativo STATISTICA para Windows 5.1B (1996).

Para avaliar os ajustes dos modelos, utilizou-se o coeficiente de determinação $\left(\mathrm{r}^{2}\right)$ e o desvio médio relativo $(\mathrm{P})$, calculado pela Equação 1. Para avaliar a significância das variações no conteúdo de umidade, com o aumento da temperatura, determinou-se o coeficiente de variação (CV) (Equação 2).

$P=\frac{100}{n} \sum_{i=1}^{n} \frac{m_{\text {exp }}-m_{\text {pre }}}{m_{\text {exp }}}$

onde: $P$ é o desvio médio relativo; $m_{\exp }$ e $m_{\text {pre }}$ as umidades experimental e predita, respectivamente; e $n$, o número de observações.

$C V=\frac{s}{m} \cdot 100$

onde: CV é o coeficiente de variação; s, o desvio padrão; e $\overline{\mathrm{m}}$, a média aritmética da umidade.

\subsection{Determinação da monocamada}

O valor da monocamada $\left(\mathrm{m}_{\mathrm{o}}\right)$ foi determinado utilizando-se a forma linearizada da equação de BET (Equação 3) (BRUNAUER; EMMET; TELLER, 1938).

$\frac{a_{w}}{\left(1-a_{w}\right) \cdot m}=\frac{1}{m_{o} \cdot C}+\frac{(C-1)}{m_{o} \cdot C} \cdot a_{w}$

Tabela 1. Modelos utilizados nos ajustes.

\begin{tabular}{cc}
\hline \multicolumn{1}{c}{ Nome } & Modelo \\
\hline Handerson (CHIRIFE; IGLESIAS, 1978) & $m=\left[\frac{-\ln \left(1-a_{w}\right)}{a}\right]^{\frac{1}{b}}$ \\
Oswin (CHIRIFE; IGLESIAS, 1978) & $m=\frac{a\left[\frac{a_{w}}{1-a_{w}}\right]^{b}}{\left[\left(1-c \cdot a_{w}\right) \cdot\left(1+(b-1) \cdot c \cdot a_{w}\right)\right]}$ \\
GAB (MAROULIS et al., 1988) & $m=\frac{a \cdot b \cdot c \cdot a_{w}}{\left[\left(1-c \cdot a_{w}\right) \cdot\left(1+(b-1) \cdot c \cdot a_{w}\right)\right]}$ \\
BET-modificado (JAAFAR; MICHALOWSKI, 1990) & $a \cdot b \cdot a_{w}$ \\
\hline
\end{tabular}

$\mathrm{m}=$ umidade ( $\mathrm{g} \mathrm{H}_{2} \mathrm{O} .100 \mathrm{~g}^{-1}$ b.s. $) ; \mathrm{a}_{\mathrm{w}}=$ atividade de água; $\mathrm{a}, \mathrm{b}$ e $\mathrm{c}=$ constantes dos modelos. 
onde: $\mathrm{m}$ é a umidade ( $\mathrm{g} \mathrm{H}_{2} \mathrm{O} .100 \mathrm{~g}^{-1}$ b.s.); $\mathrm{a}_{\mathrm{w}}$, a atividade de água; $\mathrm{m}_{\mathrm{o}}$, a umidade da monocamada $\left(\mathrm{g} \mathrm{H}_{2} \mathrm{O} .100 \mathrm{~g}^{-1}\right.$ b.s.); e C, constante relacionada com o calor de sorção.

\subsection{Determinação do calor de dessorção}

$\mathrm{O}$ calor isostérico integral de dessorção $\left(\mathrm{Q}_{\mathrm{st}}\right)$ foi determinado utilizando a Equação 4 e o calor isostérico líquido de dessorção $\left(\mathrm{q}_{\mathrm{st}}\right)$ através da Equação 5.

$Q_{s t}=q_{s t}+\Delta H_{v}$

$\frac{d\left(\ln a_{w}\right)}{d(1 / T)}=\frac{-q_{s t}}{R}$

onde: $\mathrm{Q}_{\mathrm{st}}$ é o calor isostérico integral de dessorção; $\mathrm{q}_{\mathrm{st}}$ o calor isostérico líquido de dessorção; $\Delta \mathrm{H}_{\mathrm{v}}$ a entalpia de vaporização da água $\left(\mathrm{kJ} \cdot \mathrm{mol}^{-1}\right) ; \mathrm{a}_{\mathrm{w}}$ a atividade de água; $\mathrm{T}$, temperatura absoluta $(\mathrm{K})$; e R, a constante universal dos gases $\left(\mathrm{J} \cdot \mathrm{mol}^{-1} \cdot \mathrm{K}^{-1}\right)$.

\section{Resultados e discussão}

\subsection{Caracterização físico-química da matéria-prima}

Os resultados obtidos na caracterização físico-química do açaí e do cupuaçu em pó estudados são apresentados na Tabela 2. Os produtos apresentaram uma composição aproximadamente três vezes inferior aos obtidos por Rogez (2000) e Rogez et al. (2004) para o açaí e cupuaçu in natura, em base seca; com exceção do teor de carboidratos totais, que foi superior. $\mathrm{O}$ elevado teor deste constituinte é um forte indício de que, ao contrário do informado pelo fabricante, tenha havido adição de coadjuvante nas formulações. O elevado teor deste constituinte proporcionou a redução dos teores dos demais componentes, oferecendo provavelmente produtos mais estáveis, porém com composição nutricional reduzida.

\subsection{Dados de sorção de umidade}

A Tabela 3 apresenta os dados de sorção de umidade (adsorção e dessorção), obtidos experimentalmente para os produtos estudados, nas diferentes condições de temperatura.

Com base nos dados de adsorção, constata-se que tanto para o açaí quanto para o cupuaçu em pó apresentarem níveis de $\mathrm{a}_{\mathrm{w}}$ superiores a 0,6 há necessidade de estarem com níveis de umidade superiores a $10 \mathrm{~g} \mathrm{H}_{2} \mathrm{O} .100 \mathrm{~g} \mathrm{~g}^{-1}$ b.s.; quando se leva em consideração toda a faixa de temperatura estudada. De acordo com as pesquisas de Scott (1957), 0,6 é o limite de $\mathrm{a}_{\mathrm{w}}$ abaixo do qual um alimento tem assegurada sua estabilidade microbiológica.

Tabela 2. Caracterização físico-química das amostras.

\begin{tabular}{lrc}
\multicolumn{1}{c}{ Composição $\left(\mathrm{g} .100 \mathrm{~g}^{-1} \mathrm{~b} . \mathrm{s}\right)$} & \multicolumn{1}{c}{ Açaí em pó } & Cupuaçu em pó \\
\hline Umidade & $5,04 \pm 0,05$ & $6,63 \pm 0,04$ \\
Proteínas totais $(\mathrm{N} \times 6,25)$ & $3,60 \pm 0,08$ & $2,20 \pm 0,04$ \\
Gorduras & $14,20 \pm 0,06$ & $0,80 \pm 0,02$ \\
Resíduo mineral fixo (Cinzas) & $1,30 \pm 0,01$ & $1,30 \pm 0,01$ \\
Carboidratos totais & $80,90 \pm 0,02$ & $95,70 \pm 0,05$ \\
\hline
\end{tabular}

$\mathrm{N}=$ nitrogênio total.
Nenhuma transformação visualmente perceptível, como perda de fluidez, escurecimento ou desenvolvimento de fungos, foi observada nos produtos durante os ensaios de adsorção e dessorção, na faixa de atividade de água estudada.

\subsection{Isotermas de sorção de umidade}

Nas Figuras 1 a 3, estão apresentadas as isotermas de adsorção e dessorção de umidade para o açaí em pó e, nas Figuras 4 a 6, para o cupuaçu em pó, nas temperaturas de 15,25 e $35^{\circ} \mathrm{C}$, respectivamente. Ambos os produtos apresentaram isotermas do tipo III, de acordo com a classificação de Salwin (1959) e IUPAC (1985).

As isotermas do açaí e do cupuaçu em pó apresentam um comportamento linear (comprovado através de regressão linear), com variação máxima de $5 \mathrm{~g} \mathrm{H}_{2} \mathrm{O} .100 \mathrm{~g}^{-1}$ b.s., até níveis de 0,60 e 0,65 de $a_{w}$, respectivamente. A partir destes valores, as isotermas passaram a apresentar um comportamento exponencial, com aumento progressivo da quantidade de umidade retida pelos produtos.

O comportamento observado indica que os produtos requerem maiores cuidados, quando armazenados em ambientes com umidades relativas superiores a 60 e $65 \%$, respectivamente. Nestas condições, para evitar o ganho excessivo de umidade pelos produtos, recomenda-se a utilização de embalagens que ofereçam resistência à passagem de umidade (embalagens impermeáveis ou com baixa permeabilidade ao vapor de água).

Analisando as Figuras de 1 a 6, observa-se ainda que tanto o açaí em pó quanto o cupuaçu em pó apresentaram diferença entre as isotermas de adsorção e dessorção de umidade, caracterizando o efeito de histerese. Este efeito se prolonga sobre praticamente toda a faixa de $\mathrm{a}_{\mathrm{w}}$ estudada, sendo mais pronunciado na região de $\mathrm{a}_{\mathrm{w}}$ intermediária; estando de acordo com Labuza (1968).

\subsection{Efeito da temperatura sobre as isotermas}

As Figuras 7 e 8 e as Figuras 9 e 10 ilustram o efeito da temperatura sobre as isotermas de adsorção e dessorção, para o açaí em pó e cupuaçu em pó, respectivamente.

Para as isotermas de adsorção e dessorção, a variação média da umidade em função da temperatura; determinada através do coeficiente de variação $(\mathrm{CV})$, em toda faixa de $\mathrm{a}_{\mathrm{w}}$ estudada, foi de 8 e $10 \%$ para o açaí em pó, e de 1 e $5 \%$ para o cupuaçu em pó, respectivamente. Considerando como valor limite 5\% (GOMES, 1984), as isotermas de dessorção do cupuaçu em pó são as únicas que se encontram dentro da faixa de variabilidade limitante (5\%). Pode-se considerar, desta forma, que a temperatura não exerceu influência significativa sobre as isotermas de dessorção de umidade do cupuaçu em pó, pois a variabilidade observada pode ser atribuída exclusivamente a erros experimentais e aos ajustes matemáticos.

\subsection{Predição das isotermas de sorção}

Na Tabela 4, encontram-se os valores dos coeficientes de determinação $\left(\mathrm{r}^{2}\right)$ e desvio médio relativo $\mathrm{P}(\%)$, referentes aos 
Tabela 3. Dados de sorção de umidade para o açaí e cupuaçu em pó.

\begin{tabular}{|c|c|c|c|c|c|c|c|c|c|c|c|c|}
\hline & \multicolumn{4}{|c|}{$15^{\circ} \mathrm{C}$} & \multicolumn{4}{|c|}{$25^{\circ} \mathrm{C}$} & \multicolumn{4}{|c|}{$35^{\circ} \mathrm{C}$} \\
\hline & \multicolumn{2}{|c|}{ Adsorção } & \multicolumn{2}{|c|}{ Dessorção } & \multicolumn{2}{|c|}{ Adsorção } & \multicolumn{2}{|c|}{ Dessorção } & \multicolumn{2}{|c|}{ Adsorção } & \multicolumn{2}{|c|}{ Dessorção } \\
\hline & $a_{w}$ & $\mathrm{~m}$ & $a_{w}$ & $\mathrm{~m}$ & $a_{w}$ & $\mathrm{~m}$ & $a_{w}$ & $\mathrm{~m}$ & $a_{w}$ & $\mathrm{~m}$ & $a_{w}$ & $\mathrm{~m}$ \\
\hline \multirow[t]{12}{*}{ Açaí } & 0,13 & 5,80 & 0,96 & 30,18 & 0,11 & 5,59 & 0,93 & 29,95 & 0,10 & 3,93 & 0,93 & 26,66 \\
\hline & 0,17 & 6,49 & 0,83 & 18,02 & 0,23 & 6,70 & 0,90 & 27,54 & 0,13 & 4,30 & 0,91 & 23,31 \\
\hline & 0,25 & 7,26 & 0,81 & 17,47 & 0,37 & 7,57 & 0,88 & 26,06 & 0,20 & 5,36 & 0,87 & 20,17 \\
\hline & 0,36 & 8,36 & 0,78 & 16,78 & 0,43 & 8,04 & 0,82 & 19,46 & 0,26 & 6,20 & 0,82 & 17,06 \\
\hline & 0,46 & 9,35 & 0,73 & 15,98 & 0,55 & 9,33 & 0,78 & 17,22 & 0,45 & 7,78 & 0,77 & 15,12 \\
\hline & 0,54 & 10,81 & 0,69 & 15,20 & 0,58 & 10,15 & 0,67 & 14,55 & 0,54 & 9,21 & 0,61 & 12,25 \\
\hline & 0,59 & 11,57 & 0,66 & 14,83 & 0,67 & 12,42 & 0,50 & 12,24 & 0,64 & 11,08 & 0,52 & 11,03 \\
\hline & 0,65 & 12,55 & 0,58 & 13,70 & 0,77 & 15,38 & 0,42 & 11,08 & 0,73 & 12,93 & 0,43 & 9,78 \\
\hline & 0,76 & 14,85 & 0,47 & 12,48 & 0,79 & 16,71 & 0,35 & 10,33 & 0,80 & 15,19 & 0,35 & 9,65 \\
\hline & 0,83 & 18,57 & 0,43 & 11,98 & 0,84 & 19,84 & 0,26 & 9,17 & 0,84 & 17,36 & 0,31 & 8,73 \\
\hline & 0,84 & 19,12 & 0,23 & 8,92 & 0,90 & 26,03 & 0,20 & 8,47 & 0,91 & 23,75 & 0,25 & 7,96 \\
\hline & 0,96 & 30,18 & 0,14 & 7,33 & 0,93 & 29,95 & 0,12 & 6,59 & 0,93 & 26,66 & 0,14 & 5,75 \\
\hline \multirow[t]{12}{*}{ Cupuaçu } & 0,18 & 5,22 & 0,94 & 64,94 & 0,13 & 4,83 & 0,94 & 45,97 & 0,10 & 4,64 & 0,95 & 51,78 \\
\hline & 0,37 & 6,42 & 0,88 & 35,34 & 0,16 & 4,84 & 0,86 & 26,39 & 0,15 & 5,07 & 0,89 & 32,50 \\
\hline & 0,46 & 6,98 & 0,85 & 28,75 & 0,27 & 5,26 & 0,80 & 22,05 & 0,30 & 6,35 & 0,87 & 29,21 \\
\hline & 0,49 & 7,42 & 0,83 & 26,35 & 0,35 & 6,23 & 0,77 & 20,05 & 0,36 & 6,74 & 0,84 & 26,22 \\
\hline & 0,54 & 8,36 & 0,81 & 24,71 & 0,49 & 7,74 & 0,75 & 18,09 & 0,44 & 7,99 & 0,76 & 19,21 \\
\hline & 0,58 & 10,08 & 0,76 & 20,09 & 0,58 & 10,51 & 0,66 & 14,21 & 0,54 & 10,69 & 0,68 & 14,95 \\
\hline & 0,67 & 13,44 & 0,69 & 16,80 & 0,64 & 12,91 & 0,62 & 13,74 & 0,66 & 13,85 & 0,61 & 12,29 \\
\hline & 0,75 & 18,84 & 0,59 & 12,49 & 0,72 & 16,06 & 0,52 & 10,97 & 0,69 & 15,39 & 0,40 & 9,46 \\
\hline & 0,79 & 21,04 & 0,37 & 8,29 & 0,83 & 22,37 & 0,47 & 10,27 & 0,72 & 16,55 & 0,34 & 8,28 \\
\hline & 0,80 & 22,39 & 0,36 & 7,99 & 0,87 & 28,19 & 0,40 & 9,23 & 0,89 & 32,5 & 0,25 & 7,35 \\
\hline & 0,84 & 27,47 & 0,30 & 7,22 & 0,90 & 32,64 & 0,23 & 7,62 & 0,92 & 38,34 & 0,14 & 5,5 \\
\hline & 0,94 & 64,94 & 0,27 & 6,55 & 0,94 & 45,97 & 0,08 & 5,05 & 0,95 & 51,78 & 0,11 & 5,45 \\
\hline
\end{tabular}

$\mathrm{m}=$ umidade do produto ( $\mathrm{g} \mathrm{H}_{2} \mathrm{O} .100 \mathrm{~g}^{-1}$ b.s.); $\mathrm{a}_{\mathrm{w}}=$ atividade de água (adimensional); e b.s. = base seca.

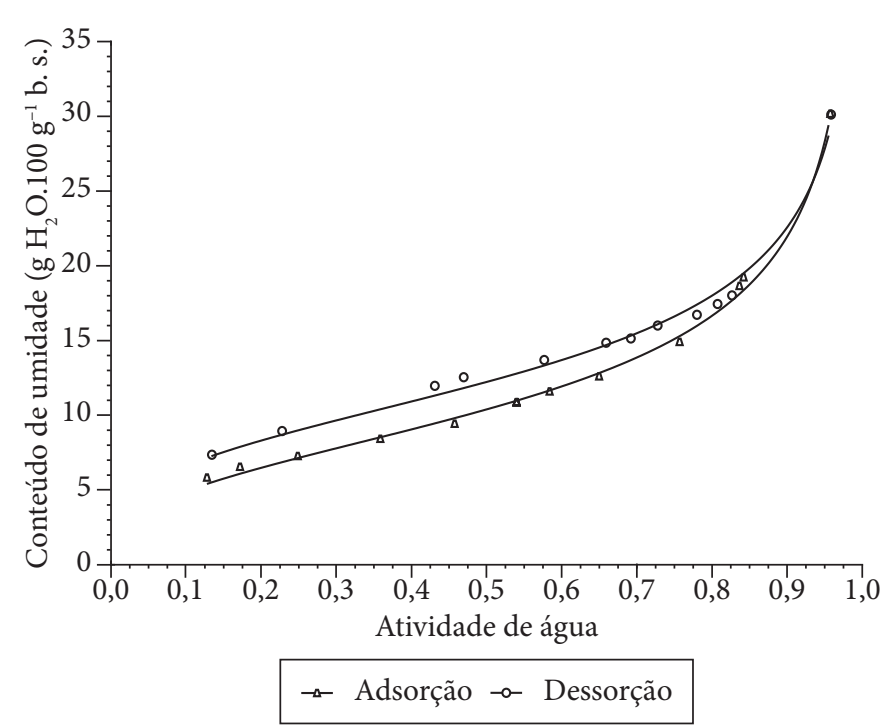

Figura 1. Isotermas de adsorção e dessorção para o açaí em pó a $15^{\circ} \mathrm{C}$.

ajustes dos modelos matemáticos testados, aos dados de adsorção e dessorção de umidade do açaí e do cupuaçu em pó.

Com base nos valores de $\mathrm{r}^{2} \mathrm{e} \mathrm{P}$, pode-se observar que os modelos de Oswin e GAB evidenciaram, de uma maneira geral, os melhores ajustes, considerando-se adsorção e dessorção para o açaí em pó e cupuaçu em pó (valor médio de $\mathrm{P}$ menor que 5\%), respectivamente. Resultados semelhantes foram encontrados

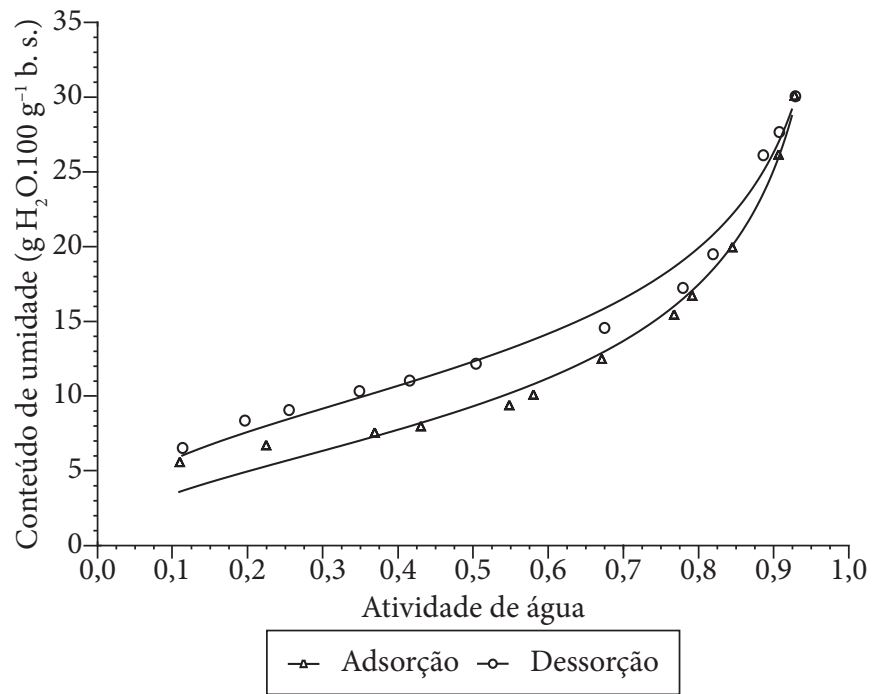

Figura 2. Isotermas de adsorção e dessorção para o açaí em pó a $25^{\circ} \mathrm{C}$.

por Park, Bin e Brod (2001) e Prado et al. (1999), em estudos realizados com diferentes frutos.

\subsection{Determinação da monocamada}

Na Tabela 5, são apresentados os valores da monocamada $\left(m_{0}\right)$, para o açaí e cupuaçu em pó, juntamente com os coeficientes de determinação $\left(\mathrm{r}^{2}\right)$, o que evidencia os bons ajustes da equação de BET. O valor médio da monocamada para o açaí em 


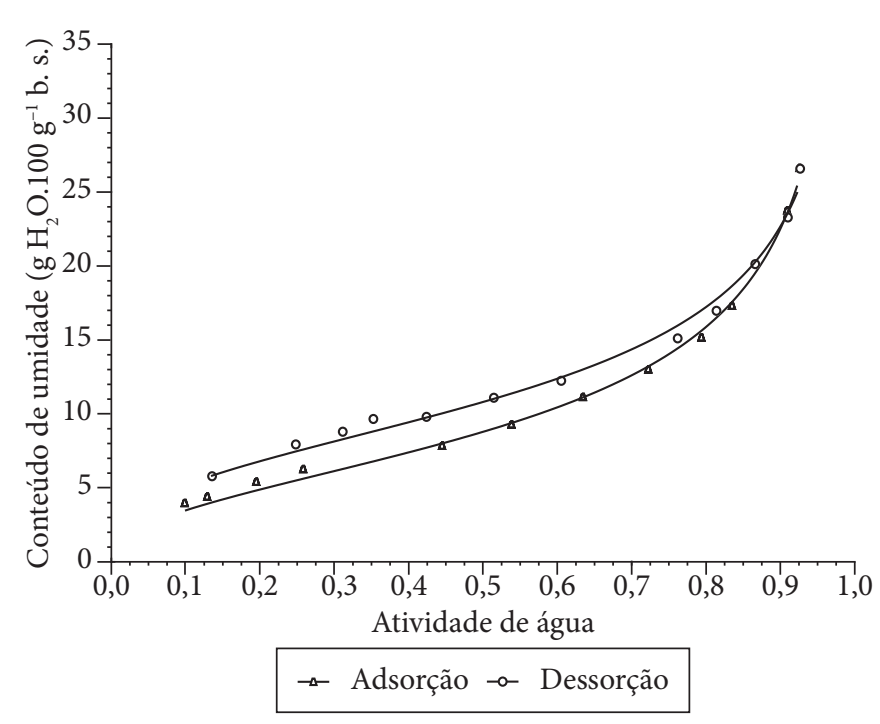

Figura 3. Isotermas de adsorção e dessorção para o açaí em pó a $35^{\circ} \mathrm{C}$.

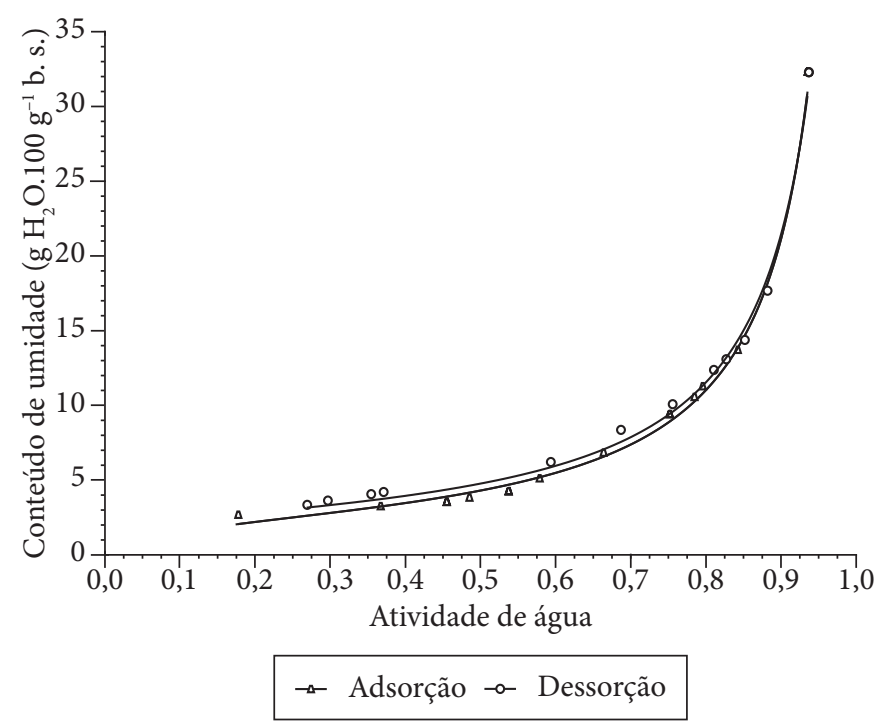

Figura 4. Isotermas de adsorção e dessorção para o cupuaçu em pó a $15^{\circ} \mathrm{C}$.

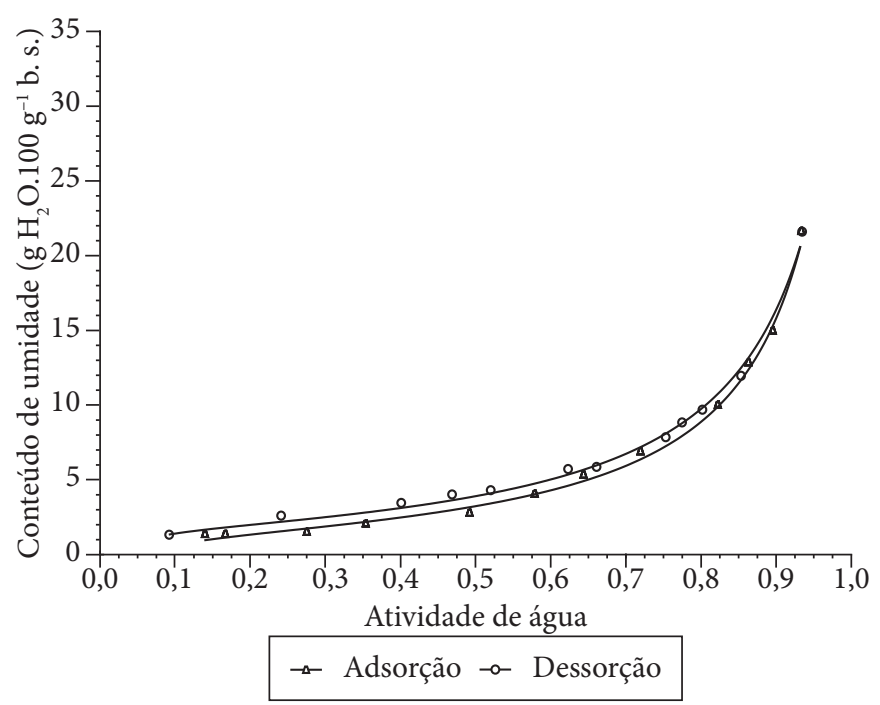

Figura 5. Isotermas de adsorção e dessorção para o cupuaçu em pó a $25^{\circ} \mathrm{C}$.

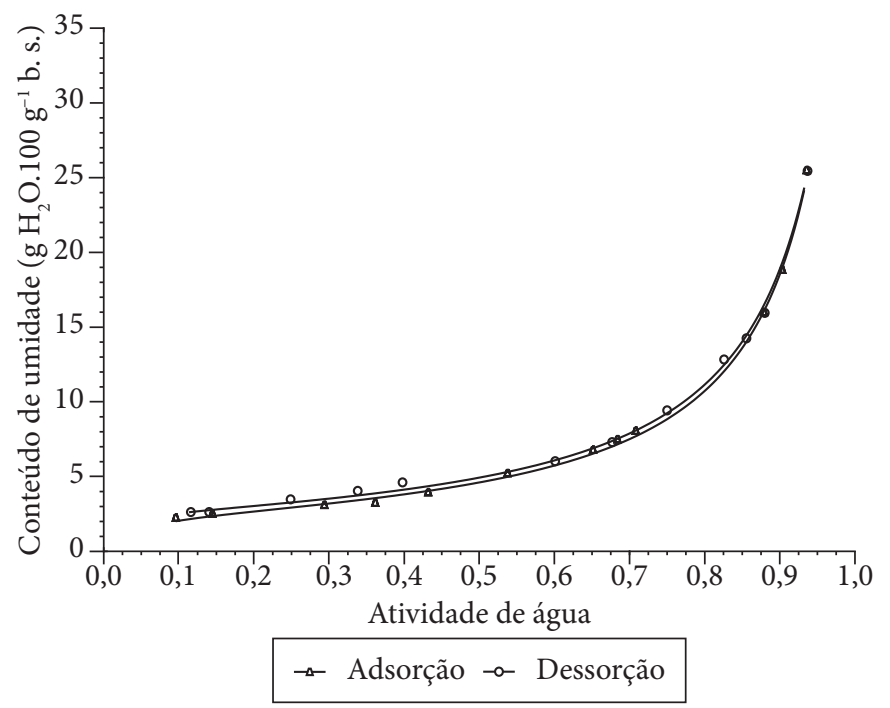

Figura 6. Isotermas de adsorção e dessorção para o cupuaçu em pó a $35^{\circ} \mathrm{C}$.

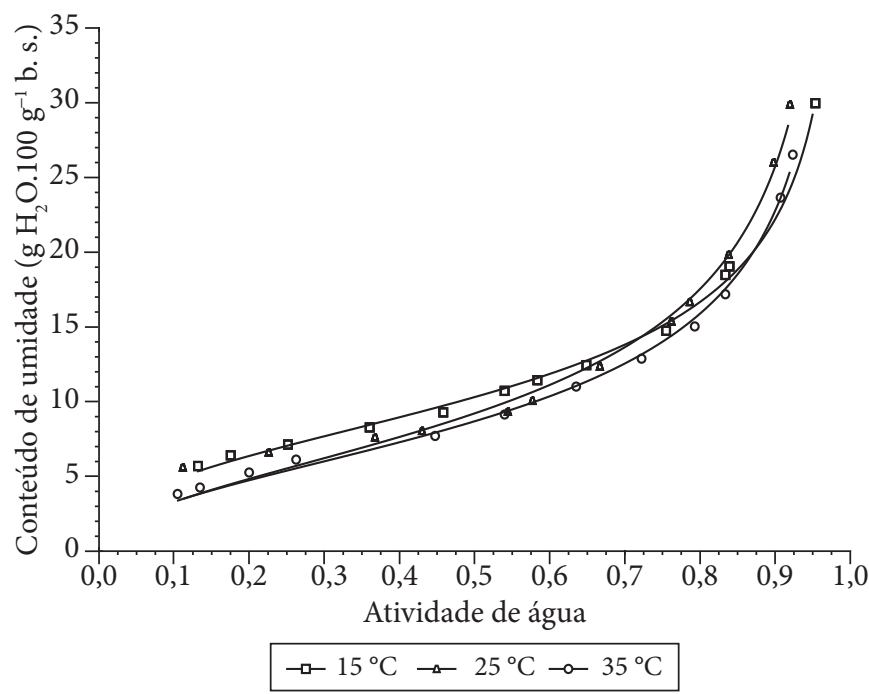

Figura 7. Efeito da temperatura nas isotermas de adsorção do açaí em pó.

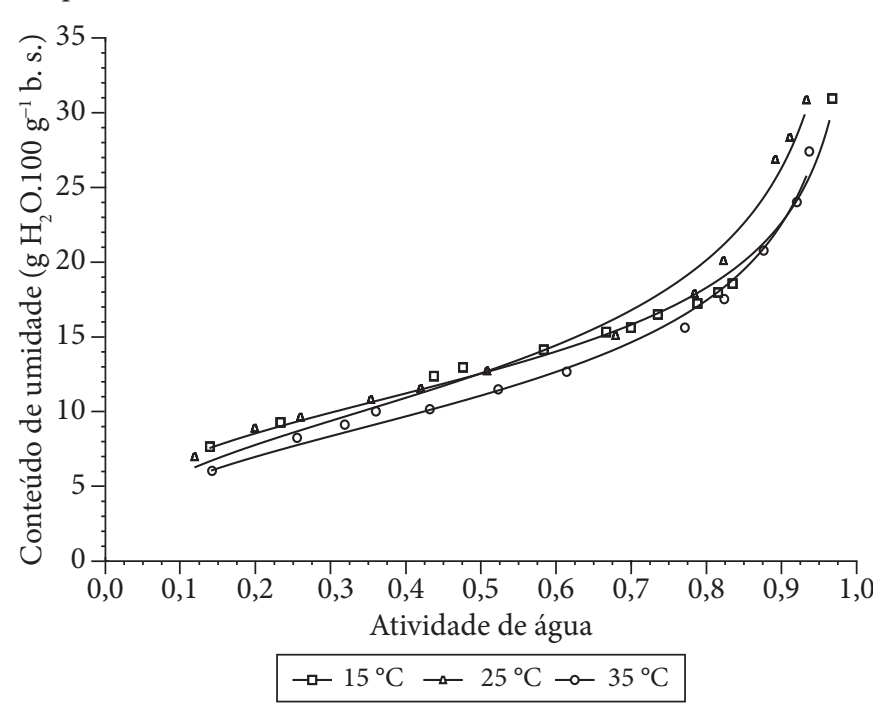

Figura 8. Efeito da temperatura nas isotermas de dessorção do açaí em pó. 


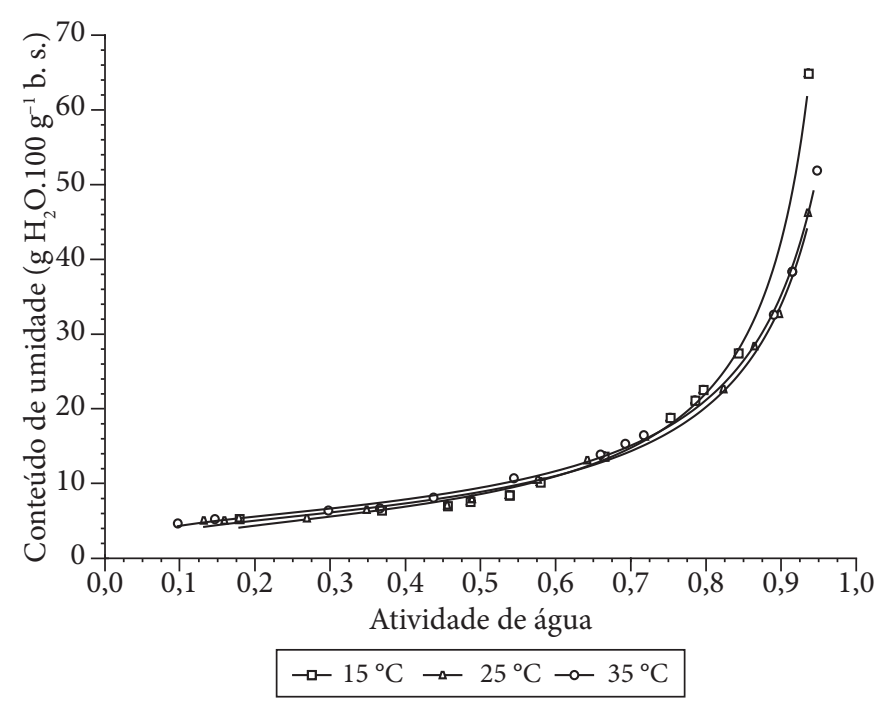

Figura 9. Efeito da temperatura nas isotermas de adsorção do cupuaçu em pó.

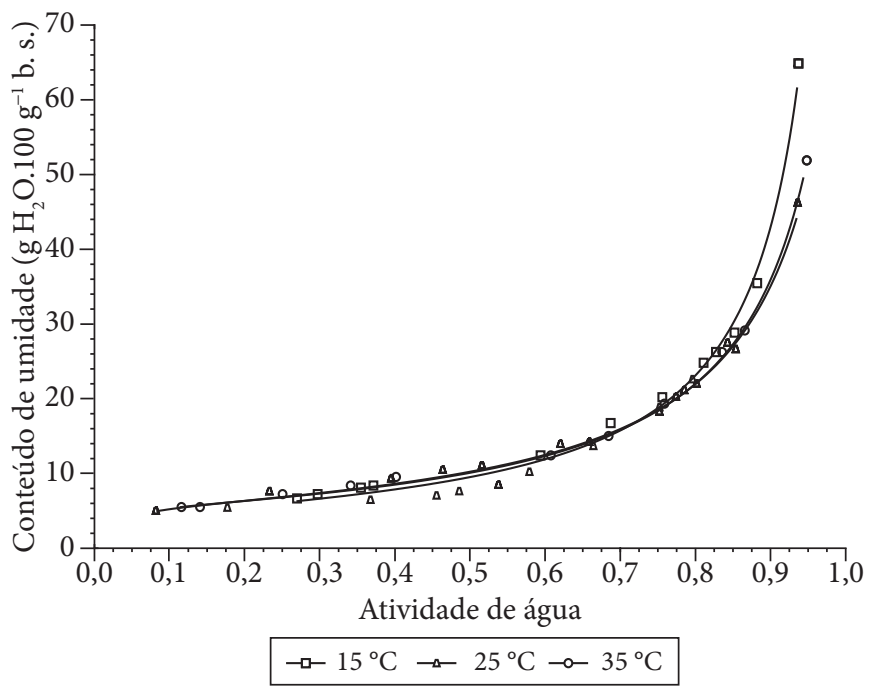

Figura 10. Efeito da temperatura nas isotermas de dessorção do cupuaçu em pó. pó foi de 7,1 $\mathrm{g} \mathrm{H}_{2} \mathrm{O} .100 \mathrm{~g}^{-1}$ b.s., e para o cupuaçu em pó de 5,6 $\mathrm{g}$ $\mathrm{H}_{2} \mathrm{O} .100 \mathrm{~g}^{-1}$ b.s., na faixa de temperatura estudada.

A monocamada é o limite de umidade abaixo do qual podem iniciar reações químicas indesejáveis e há o favorecimento da elevação do consumo de energia, para que se possa eliminar a quantidade residual de água do alimento (SALWIN, 1963).

Vale ressaltar ainda que o maior valor de $m_{o}$ para o açaí em pó indica que o produto apresenta maior concentração de constituintes com afinidade por moléculas de água que o cupuaçu em pó.

\subsection{Calor de dessorção}

Na Figura 11, é apresentado o comportamento do calor integral de dessorção $\left(Q_{s t}\right)$, em função de diferentes níveis de umidade do produto, para o açaí em pó. Observa-se que ocorre redução de $\mathrm{Q}_{\mathrm{st}}$ à medida que aumenta a umidade do produto, até atingir o valor equivalente ao calor latente de vaporização da água; nesse caso $43,4 \mathrm{~kJ} \cdot \mathrm{mol}^{-1}$.

A variação máxima observada foi pequena (menos de 40\%), em relação ao calor latente de vaporização da água, na faixa de umidade considerada (de 7,5 a 17,5 $\mathrm{g} \mathrm{H}_{2} \mathrm{O} .100 \mathrm{~g}^{-1}$ b.s.). Este comportamento é indicativo de que a umidade depende pouco da energia de interação entre as moléculas de água e dos principais solutos constituintes do açaí em pó, na faixa de temperatura estudada.

Tabela 5. Valores da monocamada para a dessorção do açaí e cupuaçu em pó.

\begin{tabular}{lcccccccc}
\hline \multirow{2}{*}{ Produto } & \multicolumn{2}{c}{$15^{\circ} \mathrm{C}$} & & \multicolumn{2}{c}{$25^{\circ} \mathrm{C}$} & & \multicolumn{2}{c}{$35^{\circ} \mathrm{C}$} \\
\cline { 2 - 5 } \cline { 7 - 8 } & $\mathrm{m}_{\mathrm{o}}$ & $\mathrm{r}^{2}$ & & $\mathrm{~m}_{\mathrm{o}}$ & $\mathrm{r}^{2}$ & & $\mathrm{~m}_{\mathrm{o}}$ & $\mathrm{r}^{2}$ \\
\hline Açaí & 6,9 & 0,9986 & & 7,7 & 0,9946 & & 6,8 & 0,9957 \\
Cupuaçu & 5,2 & 0,9943 & & 5,8 & 0,9942 & & 5,9 & 0,9975 \\
\hline
\end{tabular}

$\mathrm{r}^{2}$ = Coeficientes de determinação; e $\mathrm{P}=$ desvio médio relativo.

Tabela 4. Dados dos ajustes dos modelos aos dados de sorção dos produtos.

\begin{tabular}{|c|c|c|c|c|c|c|c|c|}
\hline & & \multirow[t]{2}{*}{ Modelo } & \multicolumn{2}{|c|}{$15^{\circ} \mathrm{C}$} & \multicolumn{2}{|c|}{$25^{\circ} \mathrm{C}$} & \multicolumn{2}{|c|}{$35^{\circ} \mathrm{C}$} \\
\hline & & & $\mathrm{r}^{2}$ & $\mathrm{P}(\%)$ & $\mathrm{r}^{2}$ & $\mathrm{P}(\%)$ & $\mathrm{r}^{2}$ & $\mathrm{P}(\%)$ \\
\hline \multirow[t]{8}{*}{ Adsorção } & Açaí & Handerson & 0,9610 & 11,7 & 0,9487 & 15,4 & 0,9637 & 15,9 \\
\hline & & Oswin & 0,9970 & 3,0 & 0,9882 & 7,5 & 0,9968 & 4,7 \\
\hline & & GAB & 0,9946 & 3,9 & 0,9958 & 4,6 & 0,9964 & 4,1 \\
\hline & & BET-modificado & 0,9948 & 3,9 & 0,9972 & 3,6 & 0,9964 & 3,9 \\
\hline & Cupuaçu & Handerson & 0,9754 & 23,2 & 0,5042 & 81,0 & 0,4769 & 88,0 \\
\hline & & Oswin & 0,9958 & 7,9 & 0,9958 & 8,3 & 0,9972 & 8,3 \\
\hline & & GAB & 0,9980 & 6,5 & 0,9976 & 5,5 & 0,9986 & 4,0 \\
\hline & & BET-modificado & 0,9980 & 6,5 & 0,9976 & 5,3 & 0,9986 & 4,0 \\
\hline \multirow[t]{8}{*}{ Dessorção } & Açaí & Handerson & 0,7916 & 8,7 & 0,9473 & 11,6 & 0,9586 & 8,8 \\
\hline & & Oswin & 0,9906 & 2,9 & 0,9860 & 5,6 & 0,9912 & 3,4 \\
\hline & & GAB & 0,9495 & 7,3 & 0,9837 & 6,4 & 0,9805 & 6,2 \\
\hline & & BET-modificado & 0,9487 & 7,5 & 0,9823 & 6,9 & 0,9765 & 6,1 \\
\hline & Cupuaçu & Handerson & 0,9444 & 28,2 & 0,3617 & 62,3 & 0,5160 & 68,8 \\
\hline & & Oswin & 0,9868 & 12,7 & 0,9904 & 8,4 & 0,9936 & 10,4 \\
\hline & & GAB & 0,9954 & 5,5 & 0,9968 & 4,0 & 0,9982 & 3,6 \\
\hline & & BET-modificado & 0,9954 & 5,9 & 0,9968 & 4,1 & 0,9982 & 3,6 \\
\hline
\end{tabular}

$\mathrm{r}^{2}=$ Coeficientes de determinação; e $\mathrm{P}=$ desvio médio relativo. 


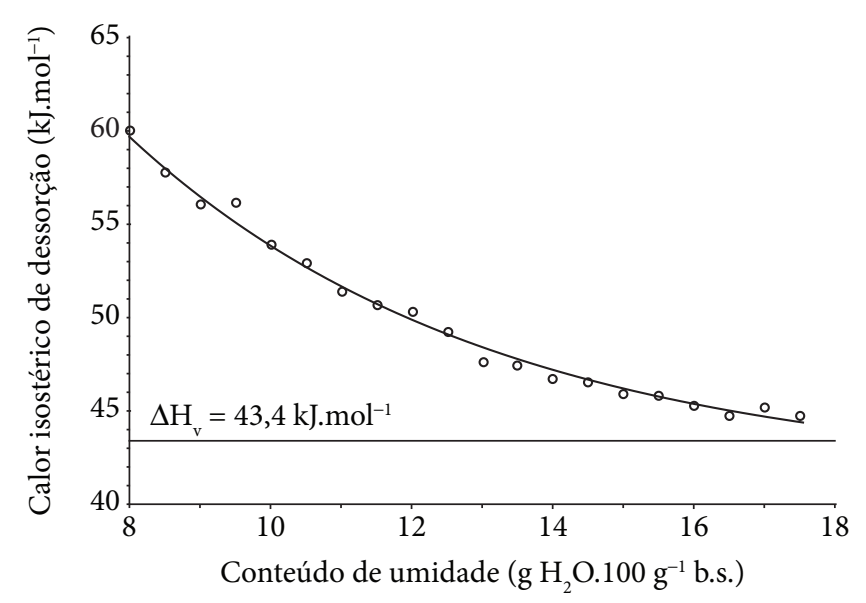

Figura 11. Calor isostérico de dessorção para o açaí em pó. $\left(\Delta \mathrm{H}_{\mathrm{v}}\right.$ - entalpia de vaporização da água).

Não foi possível determinar os calores de dessorção para o cupuaçu em pó, pois, como foi mencionado no item 3.4, as isotermas de dessorção do produto não sofrem efeito significativo da temperatura. O comportamento observado evidencia que a temperatura não interferiu na energia de interação entre as moléculas de água e dos principais solutos constituintes do cupuaçu em pó.

\section{Conclusões}

As isotermas de adsorção e dessorção para o açaí e cupuaçu em pó apresentaram um comportamento típico de isotermas do tipo III.

Os dois produtos estudados não terão asseguradas suas estabilidades microbiológicas se apresentarem níveis de umidade superiores a $10 \mathrm{~g} \mathrm{H}_{2} \mathrm{O} .100 \mathrm{~g}^{-1}$ b.s.

O açaí e o cupuaçu em pó estudados necessitarão ser devidamente acondicionados quando armazenados em ambiente com umidade relativa superior a 65\%, pois, acima deste nível, adsorverão água com velocidade cada vez maior. Assim, recomenda-se o uso de embalagens impermeáveis ou com baixa permeabilidade ao vapor de água, como as embalagens com filmes metálicos.

Os modelos de Oswin e GAB podem ser utilizados com muito boa precisão na predição dos dados de adsorção e dessorção do açaí em pó e do cupuaçu em pó, respectivamente.

A variação do calor de dessorção para o açaí em pó é um forte indício de que o produto não é altamente higroscópico, tendo o produto apresentado um valor médio para a monocamada de $7,1 \mathrm{~g} \mathrm{H}_{2} \mathrm{O} .100 \mathrm{~g}^{-1}$ b.s.

\section{Referências bibliográficas}

AOAC. ASSOCIATION OF OFFICIAL ANALYTICAL CHEMISTS. Official methods of Analysis. 16 ed. Gaithersburg, Maryland: AOAC, 1997. 1298 p.
ASSUNÇÃO, A. B.; PENA, R. S. Comportamento higroscópico do resíduo seco de camarão-rosa. Ciência e Tecnologia de Alimentos, v. 27, n. 4, p. 786-793, 2007.

BRUNAUER, S.; EMMET, P. H.; TELLER, E. Adsorpition of gases in multimolecular layers. Journal American Chemical Sociaty, v. 60, n. 2, p. 309-319, 1938.

CHIRIFE, J.; IGLESIAS, H. A. Equations for fitting water sorption isothermas of foods: Part 1 - a review. Journal Food Technology, v. 13, n. 3, p. 159-174, 1978.

GOMES, F. P. Estatística moderna da pesquisa agropecuária. Piracicaba: Potafos, 1984. 160 p.

GUERRA, R. B.; NEVES, E. C.; PENA, R. S. Caracterização e processamento de leite bubalino em pó em secador por nebulização. Ciência e Tecnologia de Alimentos, v. 25, n. 3, p. 443-447, 2005.

IBGE. INSTITUTO BRASILEIRO DE GEOGRAFIA E ESTATÍSTICA. Levantamento Sistemático da Produção Agrícola do Estado do Pará. 2006. Disponível em: http://www.ibge.gov.br/home/ estatistica/indicadores/agropecuaria/lspa/default.shtm. Acesso em: 14 março 2008.

IUPAC. INTERNATIONAL UNION OF PURE AND APPLIES CHEMISTRY. Reporting physisorption data for gas/solid systems. Pure and Applied Chemistry, v. 57, n. 4, p. 603-619, 1985.

JAAFAR, F.; MICHALOWSKI, S. Modified BET equation for sorption/ desorption isotherms. Drying Technology, v. 8, n. 4, p. 811-827, 1990.

LABUZA, T. P. Sorption phenomena in foods. Food Technology, v. 22, n. 3, p. 15-24, 1968.

MAROULIS, Z. B. et al. Application of the GAB model to the sorption isotherms for dried fruits. Journal of Food Engeneering, v. 7, n. 1, p. 63-78, 1988.

PARK, K. J.; BIN, A.; BROD, F. P. R. Obtenção das isotermas de sorção e modelagem matemática para a Pêra Bertlett Pyrus sp. com e sem desidratação osmótica. Ciência e Tecnologia de Alimentos, v. 21, n. 1, p. 73-77, 2001.

PRADO, M. E. T. et al. Isotermas de sorção de Tâmaras: determinação experimental e avaliação de modelos matemáticos. Ciência e Tecnologia de Alimentos, v. 19, n. 1, p. 143-146, 1999.

ROCKLAND, L. B. A new treatment of hygroscopic equilibria: application to walnuts (Juglans regia) and other foods. Food Research, v. 22, n. 2, p. 604-628, 1957.

ROGEZ, H. Açaí: Preparo, Composição e Melhoramento da Conservação. Belém: EDUFPA, 2000.

ROGEZ, H. et al. Chemical composition of the pulp of three typical Amazonian fruits: araça-boi (Eugenia stipitata), bacuri (Platonia insignis), cupuassu (Theobroma grandiflorum). Europe Food Research Technology, v. 218, n. 4, p. 380-384, March, 2004.

SALWIN, H. Defining minimum moisture contents for dehydrated foods. Food Technology, v. 13, n. 10, p. 594-585, 1959.

SALWIN, H. Moisture levels required for stability in dehydrated foods. Food Technology, v. 17, n. 9, p. 1114-1121, 1963.

SCOTT, W. J. Water relations of food spoilage microorganisms. Advents in Food Research, v. 7, n. 9, p. 83-127, 1957.

STATISTICA for Windows 5.1B. Computer program manual. Tulsa: StatSoft, Inc., 1996. 